\title{
On hydromagnetic rotating flow of a dusty fluid near a pulsating plate
}

\author{
SANCHITA GHOSH ${ }^{1}$ and ARUN K. GHOSH ${ }^{2}$ \\ ${ }^{1}$ Department of Computer Science, BIT Mesra, Ranchi 835215, India \\ ${ }^{2}$ Department of Mathematics, Jadavpur University, Kolkata, 700032, India \\ E-mail: akg10143@yahoo.co.in
}

\begin{abstract}
An initial value problem is solved for the motion of an incompressible viscous conducting fluid with embedded small inert spherical particles bounded by an infinite rigid nonconducting plate. Both the plate and the fluid are in a state of solid-body rotation with constant angular velocity about an axis normal to the plate. The unsteady flow is generated in the fluidparticle system due to velocity tooth pulses subjected on the plate in presence of a transverse magnetic field. It is assumed that no external electric field is imposed on the system and the magnetic Prandtl number is very small. The operational method is used to derive exact solutions for the fluid and the particle velocities and the shear stress at the wall. Some limiting cases of these solutions including the steady-state results are discussed. The general solutions for the fluid velocity and the wall shear stress are examined numerically and the simultaneous effects of rotation, the magnetic field and the particles on them are determined. Finally, the present result for the fluid velocity has been compared numerically with that generated by an impulsively moved plate in a particular case when time is large.
\end{abstract}

Mathematical subject classification: $76 \mathrm{U} 05$.

Key words: hydromagnetic, pulsatile flow, rotating dusty fluid.

\section{Introduction}

The fluid flow generated by pulsatile motion of the boundary is found to have immense importance in aerospace science, nuclear fusion, astrophysics, atmospheric sciences, cosmical gasdynamics, geophysics, and physiological fluid 
dynamics. The investigation in this direction was initiated by Ghosh [1] who examined the motion of an incompressible viscous fluid in a channel bounded by two rigid coaxial cylinders when the inner cylinder is set in motion by pulses of longitudinal impulses. Subsequently, Chakraborty and Ray [2] studied the unsteady magnetohydrodynamic Couette flow between two parallel plates when one of the plates is subjected to random pulses. Makar [3] presented the solution of magnetohydrodynamic flow between two parallel plates when the velocity tooth pulses are imposed on the upper plate and the induced magnetic field is neglected. Bestman and Njoku [4] constructed the solution of the same problem as that of author [3] without ignoring the effect of induced magnetic field. Regarding pulsatile motion of a two-phase fluid-particle system, Datta et al. [5,6] examined the heat transfer to pulsatile flow of a dusty fluid in pipes and channels with a view to their applications in the analysis of blood flow. Ghosh and Sarkar [7] considered the hydromagnetic channel flow of a dusty fluid induced by velocity tooth pulses and arrived at the solution by the method of Fourier analysis while the same problem as that of authors [7] was solved by Ghosh and Debnath [8] using the method of Laplace transforms. It is seen that both the methods give the same result although one more initial condition is needed to apply the method of Fourier analysis. Most recently, Ghosh and Ghosh [9] solved the same problem as that of authors $[7,8]$ replacing the boundary condition at the upper plate of the channel by rectified sine pulses in stead of tooth pulses and obtained the solution by using the method of Fourier analysis which appears to be simpler than that of Laplace transforms involving complicated inversions. Ghosh and Ghosh [10] also solved the hydromagnetic flow of a two-phase fluid near a pulsating plate with a view to its application in the analysis of suspension boundary layers. On the other hand, Ghosh and Debnath [11,12] have discussed the hydromagnetic Stokes flow of a rotating fluid with suspended small particles in one paper and in the other they provided solution of hydromagnetic rotating flow of a two-phase fluid-particle system in a channel bounded by two parallel plates when one of the plates is set in accelerated motion impulsively from rest. The objective of the present paper is to examine the same problem as that of authors [10] in a rotating system or to construct solution of the same problem as that of authors [11] with boundary motion being changed from a single frequency harmonic 
oscillations to multiple frequency unidirectional pulsatile motion with a view to its application in the analysis of suspension boundary layers in a rotating system [13], the motion in the liquid core of the earth which is responsible for the observed maintenance and secular variation of the mean geomagnetic field [14], the development of sunspot, the solar cycle and the structure of the magnetic stars [15] and in the flow and heat transfer characteristics of a fluid film over a rotating surface which is important in a number of industrial applications such as spreading of protective coating on electronics and optical devices, lithography and ablation cooling [16].

The problem is concerned with the unsteady flow developed in a semi-infinite expanse of an incompressible electrically conducting viscous fluid containing uniformly distributed small inert spherical particles bounded by an infinite rigid non-conducting plate in presence of an external magnetic field acting transversely to the plate when both the plate and the fluid are in a state of solid-body rotation with constant angular velocity about an axis normal to the plate and the plate is subjected to velocity tooth pulses impulsively from rest. The inquiries are made about the exact solutions for the fluid and the particle velocities and the shear stress at the wall. The results are computed numerically with a view to disclose the quantitative response of various flow parameters on the components of fluid velocity and the wall shear stress. The ultimate steady-state boundary layers are discussed. The present result for the fluid velocity at large values of time has been compared numerically with that generated by impulsively moved plate. It is seen that, in presence of pulsation, particles increase the fluid velocity near the plate only when rotation is small and time is large which is not the case in absence of pulsation. Finally, many known results are derived as limiting cases of the present solutions.

\section{Mathematical formulation}

Following Saffman [17] and Ghosh and Debnath [11], the equations of unsteady motion of an incompressible electrically conducting viscous fluid with embedded identical small inert spherical particles in a rotating coordinate system under an 
external magnetic field $\mathbf{B}$ are in usual notations:

$$
\begin{aligned}
& \frac{\partial \mathbf{u}}{\partial t}+(\mathbf{u} \cdot \nabla) \mathbf{u}+2 \boldsymbol{\Omega} \times \mathbf{u}=-\frac{1}{\rho} \nabla p+v \nabla^{2} \mathbf{u} \\
&+\frac{K N}{\rho}(\mathbf{v}-\mathbf{u})+\frac{1}{\rho}(\mathbf{j} \times \mathbf{B}) \\
& m\left[\frac{\partial \mathbf{v}}{\partial t}+(\mathbf{v} \cdot \nabla) \mathbf{v}+2 \boldsymbol{\Omega} \times \mathbf{v}\right]=K(\mathbf{u}-\mathbf{v}) \\
& \nabla \cdot \mathbf{u}=0 \quad \text { and } \quad \frac{\partial N}{\partial t}+\nabla \cdot(N \mathbf{v})=0
\end{aligned}
$$

where $\mathbf{u}=\left(u_{1}, u_{2}, u_{3}\right)$ and $\mathbf{v}=\left(v_{1}, v_{2}, v_{3}\right)$ represent the velocities of the fluid and the particles respectively, $\mathrm{p}$ is the fluid pressure, $\mathrm{N}$ is the number density of the particles which are distributed uniformly in the fluid of density $\rho$ and kinematic viscosity $v, \mathrm{~m}$ is the mass of the particle, $K$ is the Stokes' resistance coefficient which for spherical particles of radius a is $6 \pi \mu a, \mathbf{j}$ is the current density, $\mathbf{B}$ is the magnetic flux density, and $\Omega$ is the angular velocity of the coordinate system.

The Maxwell equations with usual MHD approximation are:

$$
\begin{gathered}
\nabla \cdot \mathbf{B}=0, \quad \nabla \times \mathbf{B}=\mu_{0} \mathbf{j}, \quad \nabla \times \mathbf{E}=-\frac{\partial \mathbf{B}}{\partial t}, \\
\mathbf{j}=\sigma_{0}(\mathbf{E}+\mathbf{u} \times \mathbf{B})
\end{gathered}
$$

where the displacement currents are neglected, $\mu_{0}$ and $\sigma_{0}$ are constants and $\mathbf{E}$ is the electric field.

We now consider the unsteady hydromagnetic flow in a semi-infinite expanse of an incompressible electrically conducting viscous fluid with uniformly distributed small inert spherical particles bounded by an infinite rigid nonconducting plate in presence of a constant magnetic field $B_{0}$ normal to the plate at $z=0$. Both the two-phase fluid and the plate are in a state of solid-body rotation with constant angular velocity $\Omega$ about the $z$-axis normal to the plate and in this situation the plate is set in motion in its own plane impulsively from rest by imposing velocity tooth pulses periodically on it along the $x$-axis fixed in the direction of length of the plate with $y$-axis also fixed in the plate normal to its direction of motion. 


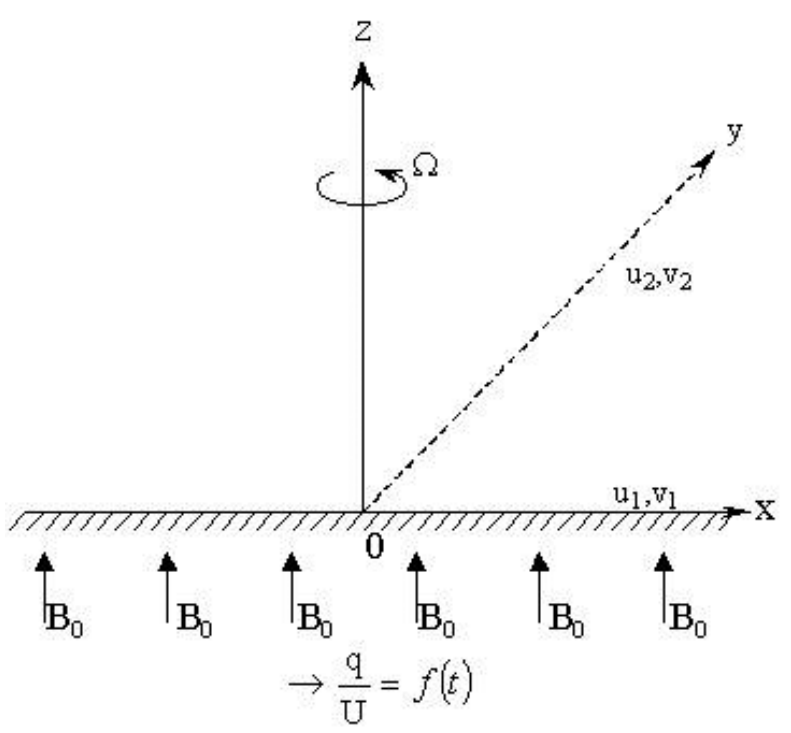

Figure 1 - Geometry of the flow configuration.

We assume that the magnetic Prandtl number $P_{m}=\sigma_{0} \mu_{0} v<<1$ which is plausible for most electrically conducting fluids and no electric field $\mathbf{E}$ is imposed on the system. This implies that the current is mainly due to induced electric field so that $\mathbf{j}=\sigma_{0}(\mathbf{u} \times \mathbf{B})$ and the applied magnetic field is essentially unaltered by the electric current flowing through the fluid. We further assume that the induced magnetic field produced by the motion of the fluid is negligible compared to the applied magnetic field so that Lorentz force term in (2.1) becomes $-\frac{\sigma_{0}}{\rho} B_{0}^{2} \mathbf{u}$. Moreover, the particles are uniformly distributed in the fluid and the flow field is parallel to the $\mathrm{x}$-direction. This implies that all the physical variables are functions of $\mathrm{z}$ and $\mathrm{t}$ only and the equation (2.3b) is satisfied throughout the flow field when $N=N_{0}=$ constant.

On the basis of the assumptions made above, the unsteady motion of a twophase fluid-particle system occupying the semi-infinite space $z>0$ is governed by the equations:

$$
\frac{\partial q}{\partial t}+2 i \Omega q=v \frac{\partial^{2} q}{\partial z^{2}}+\frac{k}{\tau}(r-q)-n q
$$


and

$$
\frac{\partial r}{\partial t}+2 i \Omega r=\frac{1}{\tau}(q-r)
$$

where $q=u_{1}+i u_{2}$ is the complex fluid velocity, and $r=v_{1}+i v_{2}$ is the complex particle velocity, $k=\frac{m N_{0}}{\rho}$ is the ratio of the mass density of the particles and the fluid density, usually called, the mass concentration of the particles, $\tau=\frac{m}{K}$ is the relaxation time of the particles and $n=\frac{\sigma_{0}}{\rho} B_{0}^{2}$ is the hydromagnetic parameter.

Introducing the non-dimensional variables

$$
\left(q^{\prime}, r^{\prime}\right)=\frac{(q, r)}{U}, \quad z^{\prime}=\frac{z}{\sqrt{v \tau}} \quad \text { and } \quad t^{\prime}=\frac{t}{\tau}
$$

and the non-dimensional flow parameters

$$
E=2 \Omega \tau, \quad n^{\prime}=n \tau
$$

in equations (2.6) and (2.7) and dropping the primes, we get the non-dimensional equations of motion in the form

$$
\frac{\partial q}{\partial t}+i E q=\frac{\partial^{2} q}{\partial z^{2}}+k(r-q)-n q
$$

and

$$
\frac{\partial r}{\partial t}+i E r=q-r .
$$

The above equations are to be solved subject to the boundary and initial conditions given by

$$
\begin{aligned}
& q(o, t)=f(t), \quad t>0, \\
& (q, r) \longrightarrow(0,0) \quad \text { as } \quad z \longrightarrow \infty, \quad t>0, \\
& (q, r)=(0,0) \quad \text { at } \quad t \leq 0 \text { for all } z
\end{aligned}
$$

where $f(t)$ represents the tooth pulses which is an even periodic function of time with period $2 T$ and strength $E_{1} T$.

\section{Solution of the problem}

In view of the nature of $f(t)$ mentioned above the mathematical form of $q(o, t)$ may be written as

$$
q(0, t)=\frac{E_{1}}{T}\left\{t H(t)+2 \sum_{p=1}^{\infty}(-1)^{p}(t-p T) H(t-p T)\right\}
$$


where $H(t)$ is the Heaviside step function defined as

$$
H(t-T)=0, t<T \quad \text { and } \quad H(t-T)=1, t \geq T .
$$

Using half-range Fourier series the condition (3.1) may also be written as

$$
q(0, t)=\frac{E_{1}}{2}-\frac{4 E_{1}}{\pi^{2}} \sum_{p=0}^{\infty} \frac{\cos [(2 p+1) \pi t / T]}{(2 p+1)^{2}} .
$$

We now use Laplace transform to the equations (2.10) and (2.11) subject to initial conditions given in $(2.14 a, b)$. The transformed equation for the fluid velocity then becomes

$$
\frac{d^{2} \bar{q}}{d z^{2}}-\left\{\frac{(S+i E+1)(S+i E+k+n)-k}{S+i E+1}\right\} \bar{q}=0
$$

with

$$
\bar{q} \longrightarrow 0 \quad \text { as } \quad z \longrightarrow \infty
$$

and

$$
\bar{q}=\frac{E_{1}}{T S^{2}} \tanh \left(\frac{S T}{2}\right) \quad \text { at } \quad z=0
$$

where $S$ is the Laplace transform variable.

The solution of (3.4) gives

$$
\bar{q}(z, S)=\frac{E_{1}}{T S^{2}} \tanh \left(\frac{S T}{2}\right) \exp \left\{-z\left[\frac{(S+i E+c)(S+i E+d)}{S+i E+1}\right]^{\frac{1}{2}}\right\}
$$

where

$$
c, d=\frac{1}{2}\left[a_{1}+n \pm\left\{a_{1}^{2}+2 n\left(a_{1}-2\right)+n^{2}\right\}^{\frac{1}{2}}\right]
$$

with $a_{1}=1+k$ and $c \geq a_{1} \geq 1>d$.

The inversion of (3.5) yields

$$
q(z, t)=\frac{E_{1}}{T} \int_{\gamma-i \infty}^{\gamma+i \infty} \frac{\tanh \left(\frac{S T}{2}\right)}{S^{2}} \exp \left\{S t-z\left[\frac{(S+i E+c)(S+i E+d)}{S+i E+1}\right]^{\frac{1}{2}}\right\} d S
$$

This inversion integral has a pole at $S=0$, a series of simple poles at $S=$ $\pm i \beta_{p}$ and branch points at $S=-(c+i E),-(1+i E),-(d+i E)$ as shown in Figure 2 in the complex S-plane where $\beta_{p}=\frac{(2 p+1) \pi}{T}, p=0,1,2, \ldots$ 


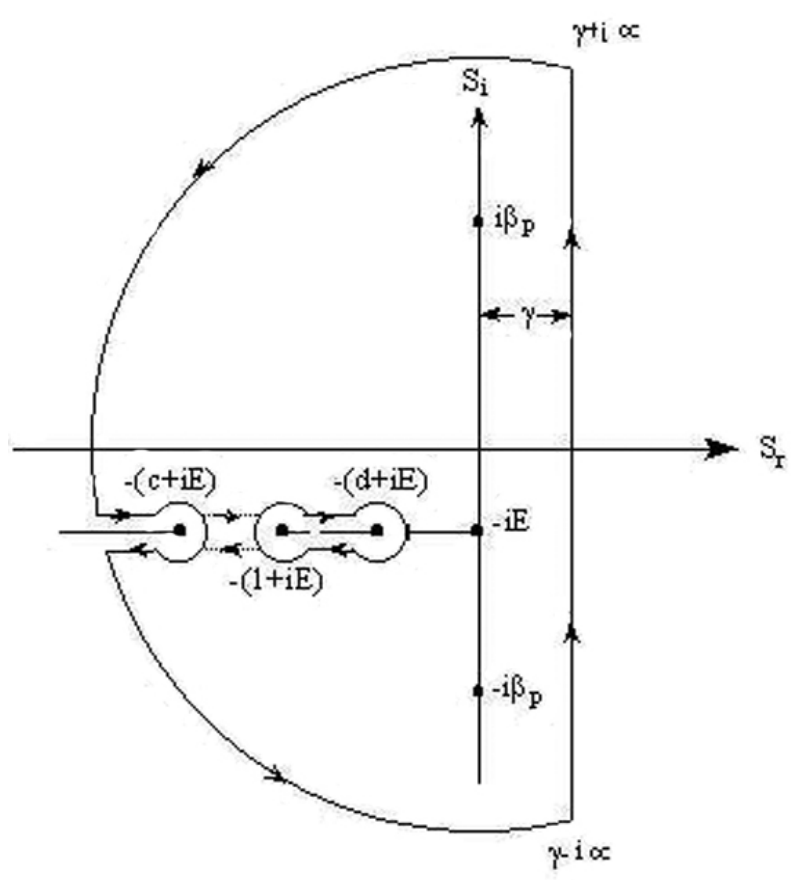

Figure 2-Contour for the integral (3.7).

On evaluation of the integral (3.7) with the help of residue theorem applied to the contour shown above, the solution for $q(z, t)$ comes out as

$$
\begin{aligned}
\frac{q(z, t)}{E_{1}}= & \frac{1}{2} \exp \left[-\frac{z}{\sqrt{2}}\left(L_{1}+i L_{2}\right)\right] \\
& -\frac{2}{T^{2}} \sum_{p=0}^{\infty} \frac{1}{\beta_{p}^{2}}\left[e^{i \beta_{p} t} \exp \left\{-\frac{z}{\sqrt{2}}\left(M_{1}+i M_{2}\right)\right\}\right. \\
& \left.+e^{-i \beta_{p} t} \exp \left\{-\frac{z}{\sqrt{2}}\left(N_{1} \pm i N_{2}\right)\right\}\right] \\
& -\frac{e^{-i E t}}{\pi T} \int_{c, d}^{\infty, 1} \frac{e^{-x t} \tanh [(x+i E) T / 2]}{(x+i E)^{2}} \\
& \times \operatorname{Sin}\left[z\left\{\frac{(x-c)(x-d)}{x-1}\right\}^{\frac{1}{2}}\right] d x
\end{aligned}
$$


where

$$
\begin{aligned}
L_{1}, L_{2}= & \left(1+E^{2}\right)^{-\frac{1}{2}}\left\{ \pm\left[c d+E^{2}(c+d-1)\right]\right. \\
& \left.+\left[\left\{c d+E^{2}(c+d-1)\right\}^{2}+E^{2}\left(c+d-c d+E^{2}\right)^{2}\right]^{\frac{1}{2}}\right\}^{\frac{1}{2}}, \quad(3.9 a, b) \\
M_{1}, M_{2}= & \left(1+\left(E+\beta_{p}\right)^{2}\right)^{-\frac{1}{2}}\left\{ \pm\left[c d+\left(E+\beta_{p}\right)^{2}(c+d-1)\right]\right. \\
& +\left[\left\{c d+\left(E+\beta_{p}\right)^{2}(c+d-1)\right\}^{2}+\left(E+\beta_{p}\right)^{2}\right. \\
& \left.\left.\times\left(c+d-c d+\left(E+\beta_{p}\right)^{2}\right)^{2}\right]^{\frac{1}{2}}\right\}^{\frac{1}{2}}, \\
N_{1}, N_{2}= & \left(1+\left(E-\beta_{p}\right)^{2}\right)^{-\frac{1}{2}}\left\{ \pm\left[c d+\left(E-\beta_{p}\right)^{2}(c+d-1)\right]\right. \\
& +\left[\left\{c d+\left(E-\beta_{p}\right)^{2}(c+d-1)\right\}^{2}+\left(E-\beta_{p}\right)^{2}\right. \\
& \left.\left.\times\left(c+d-c d+\left(E-\beta_{p}\right)^{2}\right)^{2}\right]^{\frac{1}{2}}\right\}^{\frac{1}{2}}, \\
\int_{c, d}^{\infty, 1}=\int_{c}^{\infty} & +\int_{d}^{1} \text { and } \pm \text { signs in }(3.8) \text { represent the cases } E>\beta_{p} \text { and } E<\beta_{p} \\
\text { respectively. } &
\end{aligned}
$$

The particle velocity for the corresponding motion can be determined from (2.11) as

$$
r(z, t)=e^{-(1+i E) t} \int_{0}^{t} q(z, \xi) e^{(1+i E) \xi} d \xi
$$

which on using (3.8) yields

$$
\begin{aligned}
\frac{r(z, t)}{E_{1}}= & \frac{\exp \left\{-\frac{z}{\sqrt{2}}\left(L_{1}+i L_{2}\right)\right\}\left\{1-e^{-(1+i E) t}\right\}}{2\left(1+E^{2}\right)^{\frac{1}{2}}} e^{-i \phi_{1}} \\
& -\frac{2}{T^{2}}\left[\sum_{p=0}^{\infty} \frac{\exp \left\{-\frac{z}{\sqrt{2}}\left(M_{1}+i M_{2}\right)\right\}}{\beta_{p}^{2}\left(1+\left\langle E+\beta_{p}\right\rangle^{2}\right)^{\frac{1}{2}}}\left\{e^{i \beta_{p} t}-e^{-(1+i E) t}\right\} e^{-i \phi_{2}}\right. \\
& \left.+\sum_{p=0}^{\infty} \frac{\exp \left\{-\frac{z}{\sqrt{2}}\left(N_{1} \pm i N_{2}\right)\right\}}{\beta_{p}^{2}\left(1+\left\langle E-\beta_{p}\right\rangle^{2}\right)^{\frac{1}{2}}}\left\{e^{-i \beta_{p} t}-e^{-(1+i E) t}\right\} e^{-i \phi_{3}}\right] \\
& +\frac{e^{-i E t}}{\pi T} \int_{c, d}^{\infty, 1} \frac{\tanh [(x+i E) T / 2]}{(x+i E)^{2}} \frac{e^{-x t}-e^{-t}}{x-1} \\
& \times \operatorname{Sin}\left\{z\left[\frac{(x-c)(x-d)}{x-1}\right]^{\frac{1}{2}}\right\} d x
\end{aligned}
$$


where $\tan \phi_{1}=E, \tan \phi_{2}=E+\beta_{p}$ and $\tan \phi_{3}=E-\beta_{p}$.

Thus the exact solutions for the fluid velocity $q(z, t)$ and the particle velocity $r(z, t)$ are described in equations (3.8) and (3.13) respectively.

\section{Limiting cases}

(i) The unsteady flow generated in the existing system due to impulsively moved plate can be obtained by making the time period $T \longrightarrow 0$ which in turn makes the frequency of pulsation $\beta_{p} \longrightarrow \infty$. In this situation, the modified forms of the fluid and the particle velocities, as obtained from (3.8) and (3.13) are

$$
\begin{aligned}
\frac{q(z, t)}{E_{1}}= & \frac{1}{2} \exp \left[-\frac{z}{\sqrt{2}}\left(L_{1}+i L_{2}\right)\right] \\
& -\frac{e^{-i E t}}{2 \pi} \int_{c, d}^{\infty, 1} \frac{e^{-x t}}{(x+i E)} \operatorname{Sin}\left[z\left\{\frac{(x-c)(x-d)}{x-1}\right\}^{\frac{1}{2}}\right] d x, \\
\frac{r(z, t)}{E_{1}}= & \frac{\exp \left\{-\frac{z}{\sqrt{2}}\left(L_{1}+i L_{2}\right)\right\}\left\{1-e^{-(1+i E) t}\right\}}{2\left(1+E^{2}\right)^{\frac{1}{2}}} e^{-i \phi_{1}} \\
& +\frac{e^{-i E t}}{2 \pi} \int_{c, d}^{\infty, 1} \frac{e^{-x t}-e^{-t}}{(x+i E)(x-1)} \operatorname{Sin}\left\{z\left[\frac{(x-c)(x-d)}{x-1}\right]^{\frac{1}{2}}\right\} d x .
\end{aligned}
$$

On putting $E_{1}=2$, the fluid velocity corresponding to Rayleigh problem for hydromagnetic rotating flow in a two-phase fluid can be obtained from (4.1) as

$$
\begin{aligned}
q(z, t)= & \exp \left[-\frac{z}{\sqrt{2}}\left(L_{1}+i L_{2}\right)\right] \\
& -\frac{e^{-i E t}}{\pi} \int_{c, d}^{\infty, 1} \frac{e^{-x t}}{(x+i E)} \operatorname{Sin}\left[z\left\{\frac{(x-c)(x-d)}{x-1}\right\}^{\frac{1}{2}}\right] d x,
\end{aligned}
$$

The result (4.3) is the same as that of Ghosh and Debnath [11] for their case $a=b=\frac{1}{2}, U=0$ and $\sigma=0$. In particular, when $k=0$ and $n=0$, (4.3) reduces to the result of Thornley [18] who discussed Stokes and Rayleigh layers in a rotating system. 
(ii) For the clean fluid $(k=0)$, the result (3.8) gives the fluid velocity in the form

$$
\begin{aligned}
\frac{q(z, t)}{E_{1}}= & \frac{1}{2} \exp \left[-\frac{z}{\sqrt{2}}\left(\alpha_{1}+i \alpha_{2}\right)\right] \\
& -\frac{2}{T^{2}} \sum_{p=0}^{\infty} \frac{1}{\beta_{p}^{2}}\left[\exp \left\{i \beta_{p} t-\frac{z}{\sqrt{2}}\left(m_{1}+i m_{2}\right)\right\}\right. \\
& \left.+\exp \left\{-i \beta_{p} t-\frac{z}{\sqrt{2}}\left(n_{1} \pm i n_{2}\right)\right\}\right] \\
& -\frac{e^{-i E t}}{\pi T} \int_{n}^{\infty} \frac{\tanh [(x+i E) T / 2]}{(x+i E)^{2}} \operatorname{Sin}[z \sqrt{x-n}] e^{-x t} d x
\end{aligned}
$$

where

$$
\begin{aligned}
\alpha_{1}, \alpha_{2} & =\left\{\left(n^{2}+E^{2}\right)^{1 / 2} \pm n\right\}^{1 / 2}, \\
m_{1}, m_{2} & =\left\{\left(n^{2}+\left\langle E+\beta_{p}\right\rangle^{2}\right)^{1 / 2} \pm n\right\}^{1 / 2}, \\
n_{1}, n_{2} & =\left\{\left(n^{2}+\left\langle E-\beta_{p}\right\rangle^{2}\right)^{1 / 2} \pm n\right\}^{1 / 2} .
\end{aligned}
$$

The result (4.4) is a new one and describes the fluid velocity for the hydromagnetic rotating flow of a viscous fluid near a pulsating plate.

Further, when $T \longrightarrow 0, E_{1}=2$ and $E \neq 0,(4.4)$ provides the solution of hydromagnetic Ekman problem as

$$
\begin{aligned}
q(z, t)= & \exp \left[-\frac{z}{\sqrt{2}}\left(\alpha_{1}+i \alpha_{2}\right)\right] \\
& -\frac{e^{-i E t}}{\pi} \int_{n}^{\infty} \frac{e^{-x t}}{x+i E} \operatorname{Sin}[z \sqrt{x-n}] d x .
\end{aligned}
$$

This result in the limit $n \longrightarrow 0$ yields

$$
q(z, t)=\frac{1}{2}\left[e^{z \sqrt{i E}} \operatorname{erfc}\left\langle\frac{z}{2 \sqrt{t}}+\sqrt{i E t}\right\rangle+e^{-z \sqrt{i E}} \operatorname{erfc}\left\langle\frac{z}{2 \sqrt{t}}-\sqrt{i E t}\right\rangle\right]
$$

which agrees perfectly with Thornley's [18] result (5.2) with $c=1$.

Finally, in the limit $t \longrightarrow \infty,(4.6)$ recovers the classical Ekman layer solution

$$
q(z, t)=e^{-z \sqrt{i E}}
$$


and in absence of rotation $(E=0)$, it approaches to well-known Rayleigh solution

$$
q(z, t)=\operatorname{erfc}\left(\frac{z}{2 \sqrt{t}}\right) .
$$

(iii) The solutions of the present problem in a non-rotating system $(E=0)$ are given by

$$
\begin{aligned}
\frac{q(z, t)}{E_{1}}= & \frac{1}{2} e^{-z \sqrt{c d}}-\frac{4}{T^{2}} \sum_{p=0}^{\infty} \frac{1}{\beta_{p}^{2}} e^{-\gamma_{1} z / \sqrt{2}} \operatorname{Cos}\left(\beta_{p} t-\gamma_{2} z / \sqrt{2}\right) \\
& -\frac{1}{\pi T} \int_{c, d}^{\infty, 1} \frac{e^{-x t} \tanh (x T / 2)}{x^{2}} \operatorname{Sin}\left\{z\left[\frac{(x-c)(x-d)}{x-1}\right]^{1 / 2}\right\} d x,
\end{aligned}
$$

and

$$
\begin{aligned}
\frac{r(z, t)}{E_{1}}= & \frac{1}{2} e^{-z \sqrt{c d}}\left(1-e^{-t}\right) \\
& -\frac{2}{T^{2}} \sum_{p=0}^{\infty} \frac{e^{-\gamma_{1} z / \sqrt{2}}}{\beta_{p}^{2}}\left\{\frac{e^{i \beta_{p} t}-e^{-t}}{1+i \beta_{p}} e^{-i \gamma_{2} z / \sqrt{2}}\right. \\
& \left.+\frac{e^{-i \beta_{p} t}-e^{-t}}{1-i \beta_{p}} e^{i \gamma_{2} z / \sqrt{2}}\right\} \\
& +\frac{1}{\pi T} \int_{c, d}^{\infty, 1} \frac{\tanh (x T / 2)}{x^{2}} \frac{e^{-x t}-e^{-t}}{(x-1)} \\
& \times \operatorname{Sin}\left\{z\left[\frac{(x-c)(x-d)}{x-1}\right]^{1 / 2}\right\} d x
\end{aligned}
$$

where

$$
\begin{aligned}
\gamma_{1}, \gamma_{2}= & \left(1+\beta_{p}^{2}\right)^{-1 / 2}\left\{ \pm\left[c d+\beta_{p}^{2}(c+d-1)\right]\right. \\
& \left.+\left[\left\{c d+\beta_{p}^{2}(c+d-1)\right\}^{2}+\beta_{p}^{2}\left(c+d-c d+\beta_{p}^{2}\right)^{2}\right]^{1 / 2}\right\}^{1 / 2} .
\end{aligned}
$$

The results (4.9) and (4.10) are the same as those of Ghosh and Ghosh [10]. When $T \longrightarrow 0$ and $E_{1}=2$, we get

$$
q(z, t)=e^{-z \sqrt{c d}}-\frac{1}{\pi} \int_{c, d}^{\infty, 1} \frac{e^{-x t}}{x} \operatorname{Sin}\left\{z\left[\frac{(x-c)(x-d)}{x-1}\right]^{1 / 2}\right\} d x
$$


and

$$
\begin{aligned}
r(z, t)= & e^{-z \sqrt{c d}}\left(1-e^{-t}\right)+\frac{1}{\pi} \int_{c, d}^{\infty, 1} \frac{e^{-x t}-e^{-t}}{x(x-1)} \\
& \times \operatorname{Sin}\left\{z\left[\frac{(x-c)(x-d)}{x-1}\right]^{1 / 2}\right\} d x .
\end{aligned}
$$

These results are in complete agreement with Yang and Healy [19] corresponding to their case $\omega \longrightarrow 0$ (impulsively moved plate) and describe the solutions for the fluid and the particle velocities associated with Stokes problems for a conducting fluid with suspension of particles.

Further, on putting $k=0$ (clean fluid) in (4.12), we find the fluid velocity corresponding to hydromagnetic Rayleigh problem in a non-rotating system which is mentioned in Pai [20]. The solution for the fluid velocity in this case is given by

$$
\begin{aligned}
q(z, t) & =e^{-z \sqrt{n}}-\frac{1}{\pi} \int_{n}^{\infty} \frac{e^{-x t}}{x} \operatorname{Sin}\{z \sqrt{x-n}\} d x \\
& =\frac{1}{2}\left[e^{-z \sqrt{n}} \operatorname{erfc}\langle\eta-\sqrt{n t}\rangle+e^{z \sqrt{n}} \operatorname{erfc}\langle\eta+\sqrt{n t}\rangle\right]
\end{aligned}
$$

where $\eta=\frac{z}{2 \sqrt{t}}$.

Finally, in the hydrodynamic limit $(n \rightarrow 0),(4.14)$ approaches the classical Rayleigh layer solution

$$
q(z, t)=\operatorname{erfc} \eta
$$

which is same as derived in (4.8).

(iv) The complex flow given by (3.8) and (3.13) attains the steady-state in the limit $t \rightarrow \infty$ and the ultimate flow field comprises of

$$
\begin{aligned}
\frac{q(z, t)}{E_{1}}= & \frac{1}{2} \exp \left[-\frac{z}{\sqrt{2}}\left(L_{1}+i L_{2}\right)\right] \\
& -\frac{2}{T^{2}} \sum_{p=0}^{\infty} \frac{1}{\beta_{p}^{2}}\left[e^{i \beta_{p} t} \exp \left\{-\frac{z}{\sqrt{2}}\left(M_{1}+i M_{2}\right)\right\}\right. \\
& \left.+e^{-i \beta_{p} t} \exp \left\{-\frac{z}{\sqrt{2}}\left(N_{1} \pm i N_{2}\right)\right\}\right]
\end{aligned}
$$


and

$$
\begin{aligned}
\frac{r(z, t)}{E_{1}}= & \frac{\exp \left\{-\frac{z}{\sqrt{2}}\left(L_{1}+i L_{2}\right)\right\}}{2\left(1+E^{2}\right)^{\frac{1}{2}}} e^{-i \phi_{1}} \\
& -\frac{2}{T^{2}}\left[\sum_{p=0}^{\infty} \frac{\exp \left\{i \beta_{p} t-\frac{z}{\sqrt{2}}\left(M_{1}+i M_{2}\right)\right\}}{\beta_{p}^{2}\left(1+\left\langle E+\beta_{p}\right\rangle^{2}\right)^{\frac{1}{2}}} e^{-i \phi_{2}}\right. \\
& \left.+\sum_{p=0}^{\infty} \frac{\exp \left\{-i \beta_{p} t-\frac{z}{\sqrt{2}}\left(N_{1} \pm i N_{2}\right)\right\}}{\beta_{p}^{2}\left(1+\left\langle E-\beta_{p}\right\rangle^{2}\right)^{\frac{1}{2}}} e^{-i \phi_{3}}\right]
\end{aligned}
$$

It follows from (4.16) and (4.17) that the particles in the steady-state are unable to attain the actual fluid velocity due to the presence of rotation and pulsation. But in the limit $T \rightarrow 0, E_{1}=2$ and $E=0$, we have $u_{1}=v_{1}$. This shows that, in absence of pulsation and rotation, the particles attain the fluid velocity in the steady motion generated by impulsively moved plate. This result is also known from Michael and Miller's [21] analysis.

It is also noticed from (4.16) that the ultimate flow consists of distinct multiple boundary layers, commonly known as Stokes-Ekman Hartman layers, whose thickness are of order

$$
\frac{1}{M_{1}} \sqrt{2 v \tau}, \quad \frac{1}{L_{1}} \sqrt{2 \nu \tau}, \quad \frac{1}{N_{1}} \sqrt{2 \nu \tau} \quad \text { where } \quad M_{1}>L_{1}>N_{1} .
$$

These boundary layers are modified by rotation, the magnetic field and the particles.

However, in the limit $T \rightarrow 0, E_{1}=2$ and $E \neq 0$, i.e, in the case of flow induced by impulsively moved plate in a rotating system, the above results (4.16) and (4.17) yield

$$
q(z, t)=\exp \left\{-\frac{z}{\sqrt{2}}\left(L_{1}+i L_{2}\right)\right\}
$$

and

$$
r(z, t)=\frac{\exp \left\{-\frac{z}{\sqrt{2}}\left(L_{1}+i L_{2}\right)\right\}}{\left(1+E^{2}\right)^{\frac{1}{2}}} e^{-i \phi_{1}}
$$

which are identical to those of Ghosh and Debnath [11] corresponding to their case for $\sigma=0, a=b=1 / 2$ and $U=0$. Moreover, it is noticed that, in 
absence of pulsation, all the multiple boundary layers coalesce into a single Ekman-Hartman layer of thickness of the order $\frac{1}{L_{1}} \sqrt{2 v \tau}$. In this situation, if one makes $E=0,(4.19)$ and (4.20) coincide to give

$$
q(z, t)=r(z, t)=e^{-z \sqrt{n}}
$$

which provides not only $u_{1}=v_{1}$ in a steady-state condition as stated earlier but also exhibits the existence of a single Hartman layer of thickness of order $\sqrt{v / n}$. Thus the steady-state solution of the hydromagnetic Rayleigh problem in a non-rotating system is recovered from the present analysis.

\section{The wall shear stress}

The exact solution for the components of the shear stress at the wall $z=0$ can be expressed as

$$
D_{k n E}(0, t)+i L_{k n E}(0, t)=-\left[\frac{\partial q}{\partial z}\right]_{z=0}
$$

where $D$ and $L$ stand for the drag and the Lateral stress respectively on the wall and $q$ is known from (3.8).

Substituting the expression for $q$ from (3.8) in (5.1), we get

$$
\begin{aligned}
& \frac{D_{k n E}(0, t)}{E_{1}}+i \frac{L_{k n E}(0, t)}{E_{1}} \\
& =\frac{L_{1}+i L_{2}}{2 \sqrt{2}}-\frac{\sqrt{2}}{T^{2}} \sum_{p=0}^{\infty} \frac{\left(M_{1}+i M_{2}\right) e^{i \beta_{p} t}+\left(N_{1} \pm i N_{2}\right) e^{-i \beta_{p} t}}{\beta_{p}^{2}} \\
& \quad+\frac{e^{-i E t}}{\pi T} \int_{c, d}^{\infty, 1} \frac{\tanh [(x+i E) T / 2]}{(x+i E)^{2}}\left[\frac{(x-c)(x-d)}{x-1}\right]^{1 / 2} e^{-x t} d x
\end{aligned}
$$

The corresponding steady-state result is

$$
\begin{aligned}
& \frac{D_{k n E}(0, t)}{E_{1}}+i \frac{L_{k n E}(0, t)}{E_{1}} \\
& \quad=\frac{L_{1}+i L_{2}}{2 \sqrt{2}}-\frac{\sqrt{2}}{T^{2}} \sum_{p=0}^{\infty} \frac{\left(M_{1}+i M_{2}\right) e^{i \beta_{p} t}+\left(N_{1} \pm i N_{2}\right) e^{-i \beta_{p} t}}{\beta_{p}^{2}}
\end{aligned}
$$


which clearly expresses the fact that both the drag and the lateral stress at the wall fluctuates in presence of pulsation even when the steady condition is attained and in such a situation they contain the effects of rotation, the magnetic field and the particles.

In the case of clean fluid flow $(k=0)$ and in hydrodynamic limit $(n=0)$, the result (5.2) gives

$$
\begin{aligned}
& \frac{D_{00 E}(0, t)}{E_{1}}+i \frac{L_{00 E}(0, t)}{E_{1}} \\
& =\frac{\sqrt{i E}}{2}-\frac{\sqrt{2}}{T^{2}} \sum_{0}^{\infty} \frac{\sqrt{E+\beta_{p}} e^{i\left(\beta_{p} t+\pi / 4\right)}+\sqrt{E-\beta_{p}} e^{-i\left(\beta_{p} t \mp \pi / 4\right)}}{\beta_{p}^{2}} \\
& \quad+\frac{e^{-i E t}}{\pi T} \int_{0}^{\infty} \frac{\tanh [(x+i E) T / 2]}{(x+i E)^{2}} \sqrt{x} e^{-x t} d x
\end{aligned}
$$

In this situation, if we make $T \rightarrow 0$ and put $E_{1}=2$, we get

$$
\begin{aligned}
D_{00 E}(0, t)+i L_{00 E}(0, t) & =\sqrt{i E}+\frac{e^{-i E t}}{\pi} \int_{0}^{\infty} \frac{e^{-x t}}{x+i E} \sqrt{x} d x \\
& =\sqrt{i E}(1-\operatorname{erf} c \sqrt{i E t})+\frac{1}{\sqrt{\pi t}} e^{-i E t}
\end{aligned}
$$

which describes the components of the wall shear stress for the unsteady Ekman layer flow as mentioned in Ghosh and Debnath [11]. In the limit $t \rightarrow \infty$, this result recovers the well-known steady solution

$$
D_{00 E}(0, t)+i L_{00 E}(0, t)=\sqrt{i E} .
$$

On the other hand, when $E=0,(5.2)$ yields

$$
\begin{aligned}
\frac{D_{k n 0}(0, t)}{E_{1}}= & \frac{\sqrt{c d}}{2}-\frac{2 \sqrt{2}}{T^{2}} \sum_{p=0}^{\infty} \frac{\gamma_{1} \operatorname{Cos}\left(\beta_{p} t\right)-\gamma_{2} \operatorname{Sin}\left(\beta_{p} t\right)}{\beta_{p}^{2}} \\
& +\frac{1}{\pi T} \int_{c, d}^{\infty, 1} \frac{\tanh (x T / 2)}{x^{2}}\left[\frac{(x-c)(x-d)}{x-1}\right]^{1 / 2} e^{-x t} d x
\end{aligned}
$$

which in the limit $T \rightarrow 0$ and $E_{1}=2$ gives

$$
D_{k n 0}(0, t)=\sqrt{n}+\frac{1}{\pi} \int_{c, d}^{\infty, 1}\left[\frac{(x-c)(x-d)}{x-1}\right]^{1 / 2} \frac{e^{-x t}}{x} d x .
$$


This result represents the drag on the plate exerted by a two-phase fluid under hydromagnetic situation in a non-rotating system and is the same as that given by Yang and Healy [19].

Finally, on putting $k=o$ and $n=o$, the classical viscous stress on an impulsively moved plate is obtained from (5.8) as

$$
D_{000}(0, t)=\frac{1}{\pi} \int_{0}^{\infty} \frac{e^{-x t}}{\sqrt{x}} d x=\frac{1}{\sqrt{\pi t}} .
$$

\section{Numerical results}

To investigate the effect of various flow parameters on the fluid velocity and the wall shear stress, the exact solutions (3.8) and (5.2) are evaluated for the cases $E=0, E=0.1$ and $E=1.0$ with $T=2$. These values of $E$ and $T$ are taken as typical although actual values can be used according to the physical situations. The changing nature of the velocity profiles are incorporated in Figures 3, 4(a,b) and $5(\mathrm{a}, \mathrm{b})$ for different values of the particle concentration $(k)$, the magnetic field (n) and time. It is observed from Figure 3 that in absence of rotation $(E=0)$ both the particles and the magnetic field produce diminishing effect on the fluid velocity $u_{1}$ as long as time remains small. But, at large time, a significant rise in the magnitude of the fluid velocity near the plate due to particles is noticed although the magnetic field continues to exert damping effect on it. This is a consequence of the effect of pulsation on the flow near the plate in absence of rotation.

Figure 4(a) shows that for small values of rotation, the velocity component $u_{1}$ varies in a manner similar to that of non-rotating case excepting a significant diminution in its magnitude near the plate due to rotation noticed at large values of time. It is further interesting to note that the effect of particles on $u_{1}$ near the plate at large values of time and in absence of rotation is greatly diminished with the increase of rotation. On the other hand, when rotation is small, the magnitude of the component $u_{2}$ of the fluid velocity near the plate is found to decrease due to particles and the magnetic field for small values of time and increases and decreases respectively with the particles and the magnetic field when time is large (Fig. 4(b)). The above observation shows that for small values of rotation and at large time the increasing effect of particles on $u_{1}$ component of velocity 


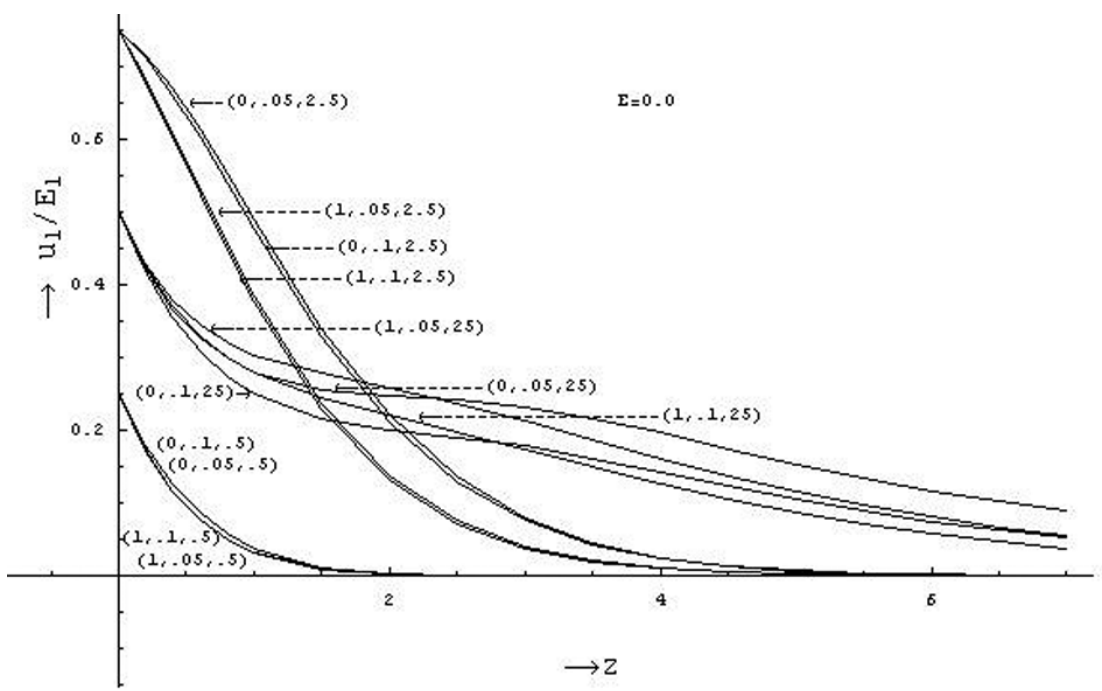

Figure 3 - Variation of the fluid velocity $u_{1}$ for different values of $(k, n, t)$ when $T=2$ and $E=0.0$.

near the plate decreases compared to non-rotating case while the $u_{2}$ component of velocity which appears due to rotation increases near the plate with the increase of particle concentration $(k)$. As a result, the fluid velocity near the plate increases with the particles only at large values of time when rotation is small. Such a phenomenon is not found when rotation is large. We therefore say that pulsation exerts its influence on the flow near the plate only when rotation is small and time is large.

When large values of rotation is considered, as shown in Figures 5(a,b), the velocity component $u_{1}$ decreases sufficiently excepting at small values of time. Moreover, $u_{1}$ becomes independent of the magnetic field for all stages of the motion when rotation is large. In this situation, the effect of particles to diminish $u_{1}$ near the plate persists even at large values of time which is not the case when rotation is small. The velocity component $u_{2}$, in the case of large rotation, increases sharply near the plate and then diminishes rapidly with $z$. This is a consequence of the effect of rotation. Besides, the magnitude of the component $u_{2}$ diminishes with particles and the magnetic field for all stages of the motion as shown in Figure 5(b). Thus when rotation is large, the pulsation cannot play a distinctive role to increase the flow near the plate at any stage of the motion. 


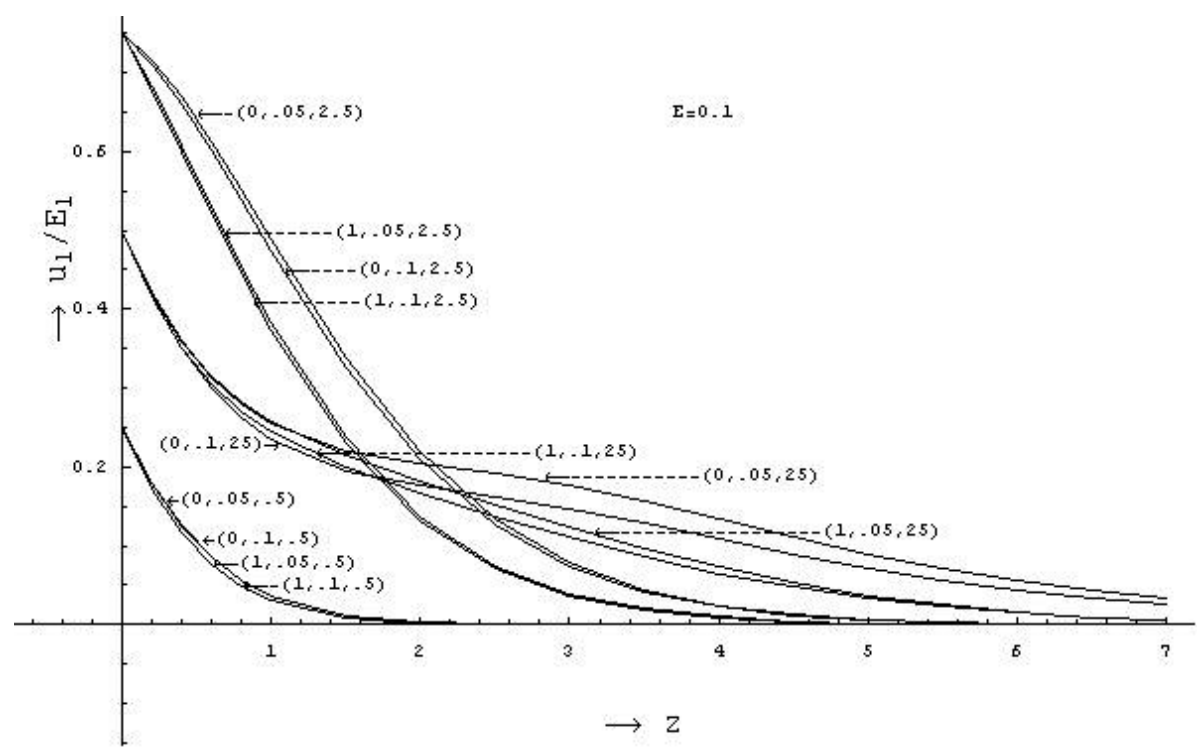

Figure 4(a) - Variation of the fluid velocity component $u_{1}$ for different values of $(k, n, t)$ when $T=2$ and $E=0.1$.

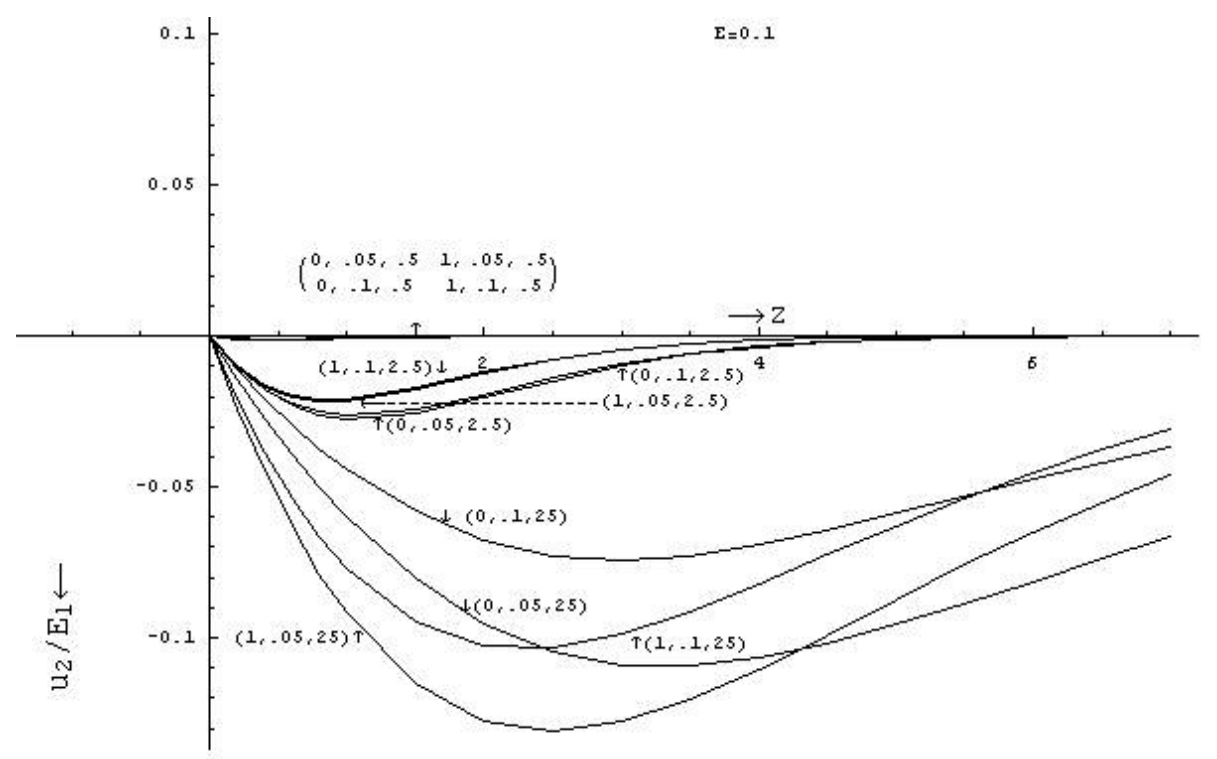

Figure 4(b) - Variation of the fluid velocity component $u_{2}$ for different values of $(k, n, t)$ when $T=2$ and $E=0.1$. 


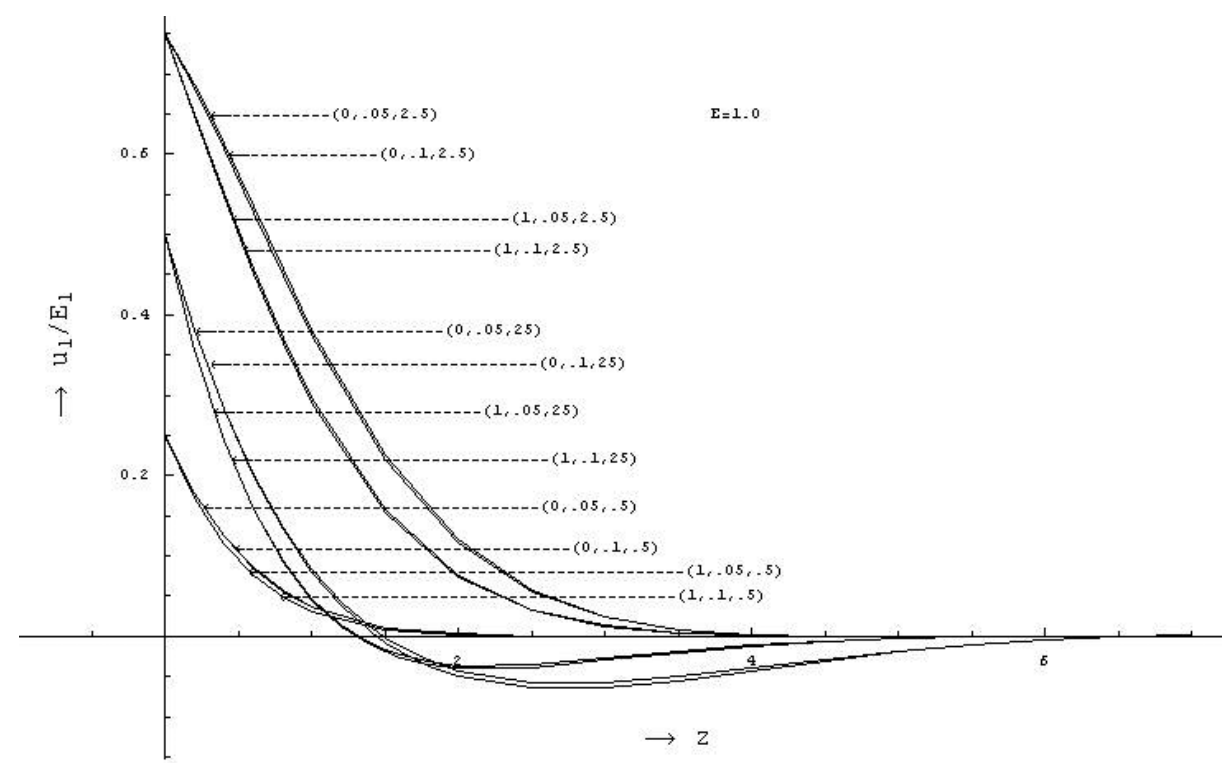

Figure 5(a) - Variation of the fluid velocity component $u_{1}$ for different values of $(k, n, t)$ when $T=2$ and $E=1.0$.

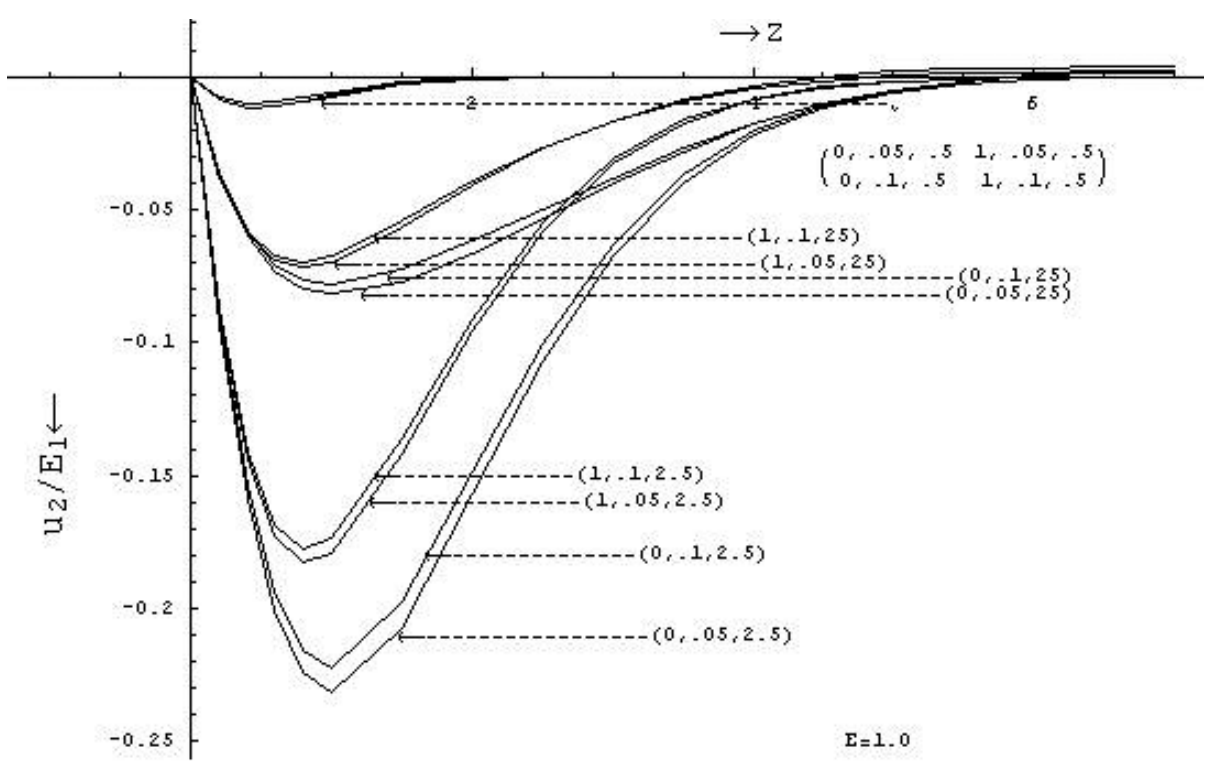

Figure 5(b) - Variation of the fluid velocity component $u_{2}$ for different values of $(k, n, t)$ when $T=2$ and $E=1.0$. 
A comparative study of the magnitude of the fluid velocity produced by pulsation of the plate has been made with that generated by impulsively moved plate for small and large values of rotation particularly when time is large. The results are presented in Figures 6,7 $(\mathrm{a}, \mathrm{b})$ and 8(a,b). The distinctive feature of this study is that the magnitude of the fluid velocity near the plate remains always higher in an impulsively generated flow compared to its value produced by pulsation of the plate. Moreover, in presence of pulsation, the increasing effect of the particles on $u_{1}$ component of fluid velocity near the plate for small rotation and large time, is totally absent in a similar situation when the flow is generated by impulsively moved plate. Additionally, the effect of pulsation on the particles to increase the fluid velocity near the plate in the case of small rotation and large time can be minimized with the increase of rotation (see Table I). In all other cases, the particles and the magnetic field produce diminishing effect on the fluid velocity near the plate for both the types of motion mentioned above.

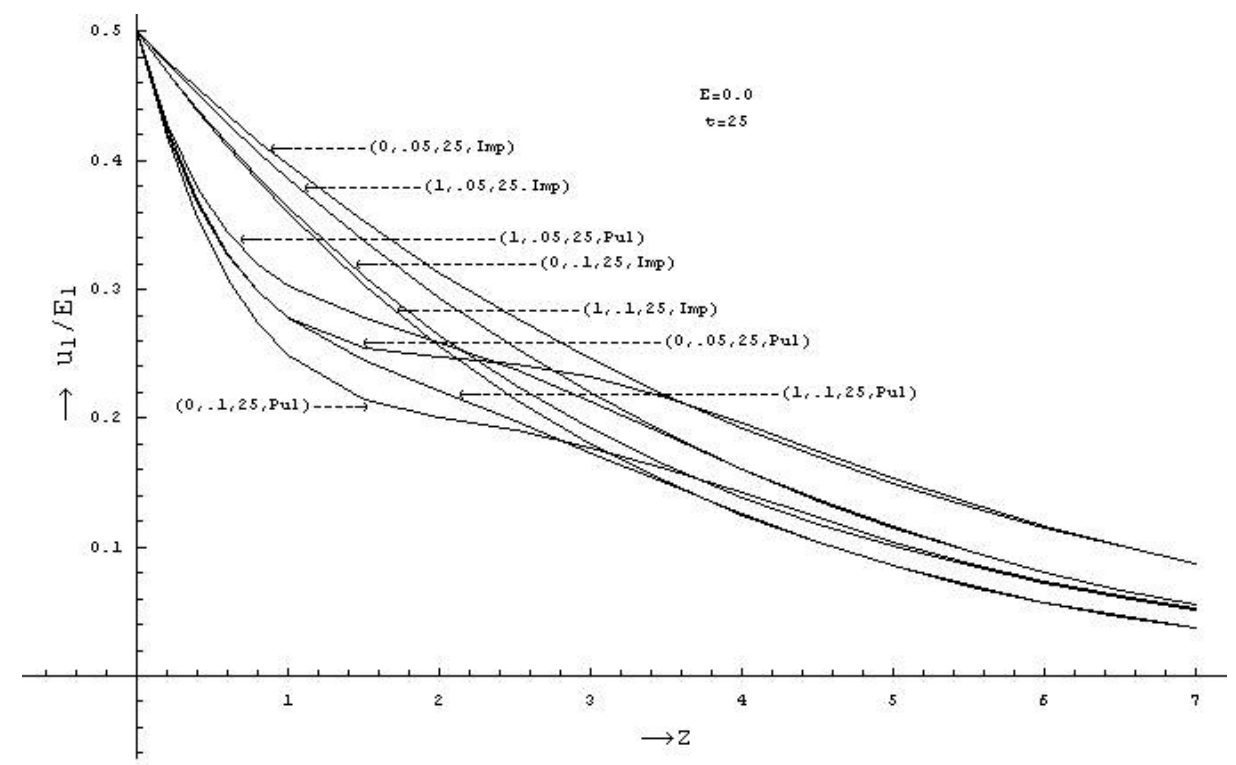

Figure 6 - Fluid velocity $u_{1}$ for different values of $(k, n)$ when $T=2, E=0.0$ and $t=25$ corresponding to both pulsatile and impulsive motion of the plate.

The distribution of drag and the lateral stress on the plate are plotted in Figures $9,10(\mathrm{a}, \mathrm{b})$ and $11(\mathrm{a}, \mathrm{b})$ with the advancement of time for the cases $E=0.0$, 


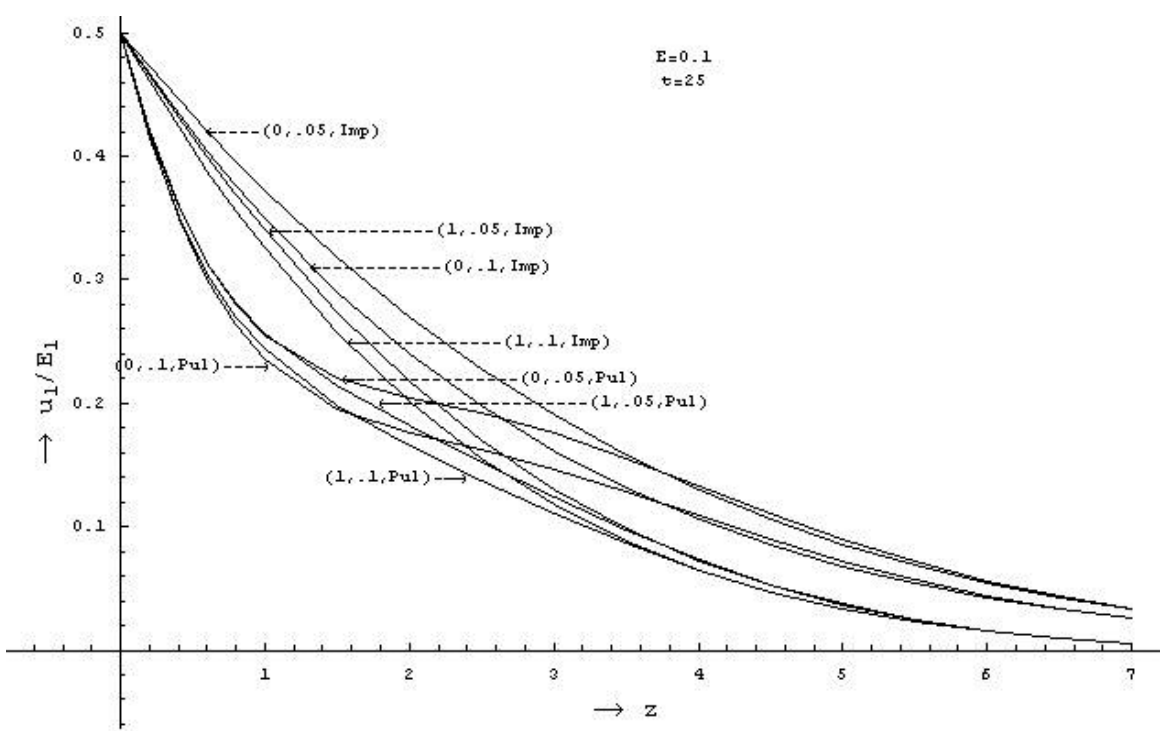

Figure 7(a) - Variation of the fluid velocity component $u_{1}$ for different values of $(k, n)$ when $T=2, E=0.1$ and $t=25$ corresponding to both pulsatile and impulsive motion of the plate.

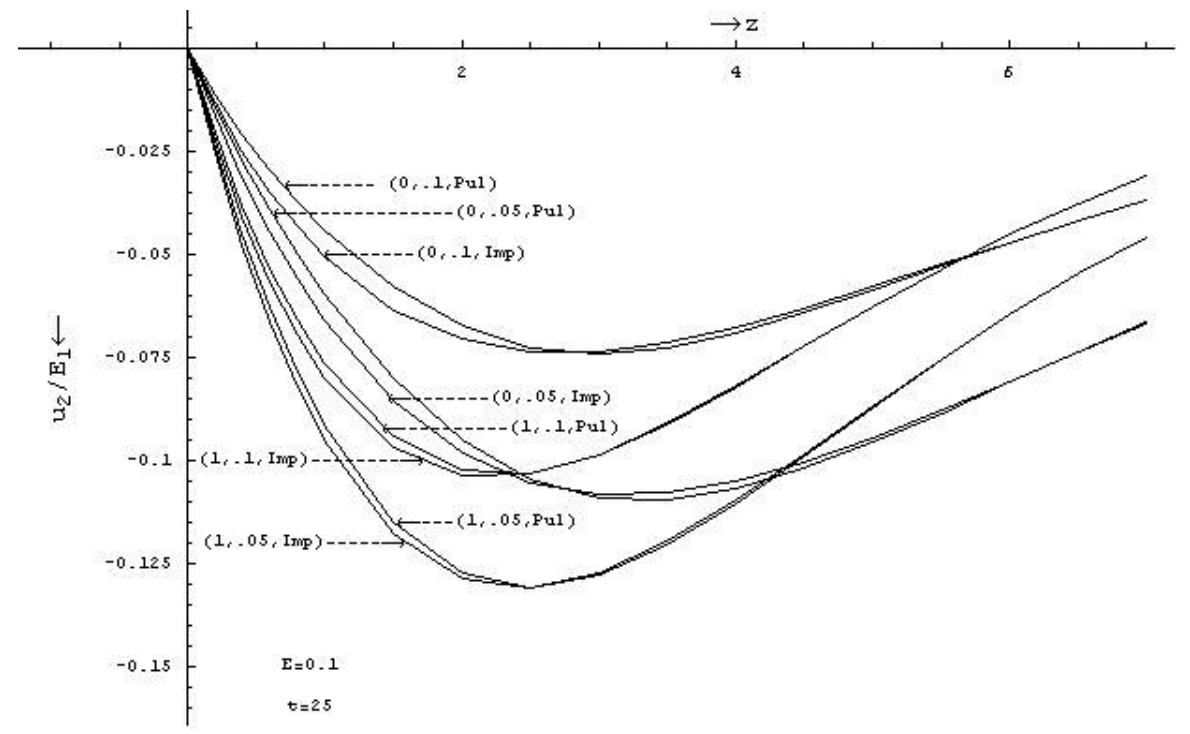

Figure 7(b) - Variation of the fluid velocity component $u_{2}$ for different values of $(k, n)$ when $T=2, E=0.1$ and $t=25$ corresponding to both pulsatile and impulsive motion of the plate. 


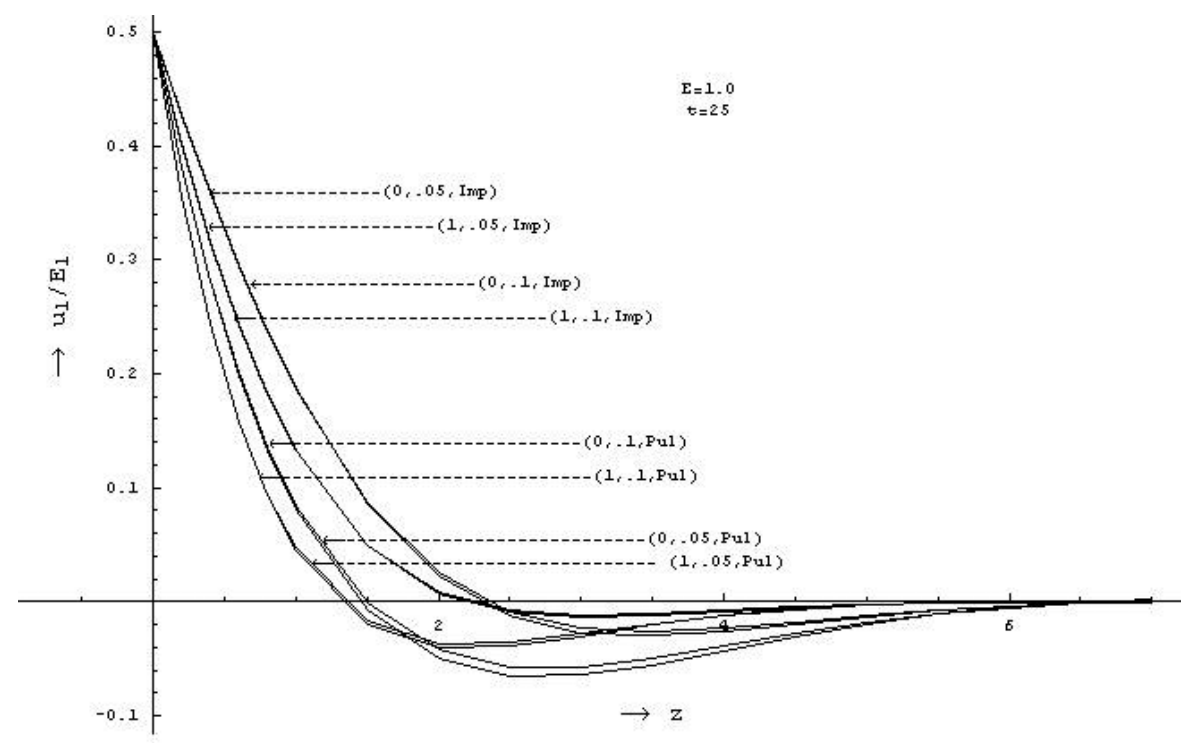

Figure 8(a) - Variation of the fluid velocity component $u_{1}$ for different values of $(k, n)$ when $T=2, E=1.0$ and $t=25$ corresponding to both pulsatile and impulsive motion of the plate.

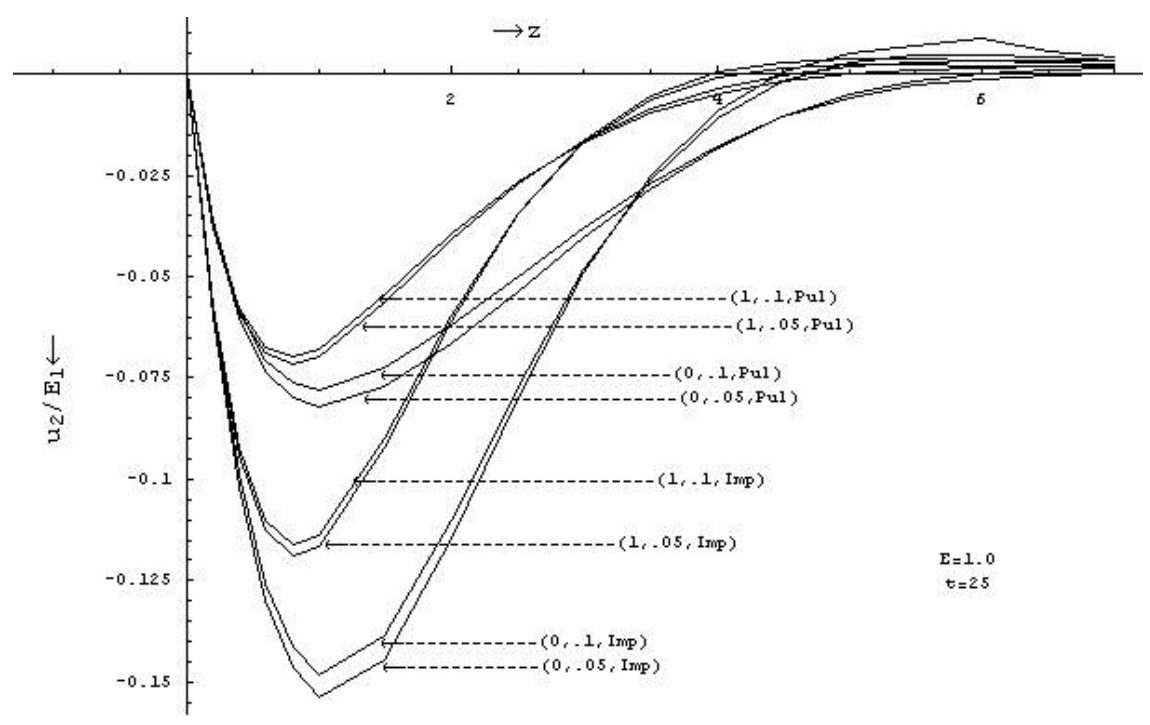

Figure 8(b) - Variation of the fluid velocity component $u_{2}$ for different values of $(k, n)$ when $T=2, E=1.0$ and $t=25$ corresponding to both pulsatile and impulsive motion of the plate. 


\begin{tabular}{|c|c|c|c|c|c|c|c|c|c|c|}
\hline $\mathrm{E}$ & $\mathrm{t}$ & $\mathrm{n}$ & $\mathrm{k}$ & $\frac{(|q|, \theta)}{z}$ & 0.0 & 0.5 & 1.0 & 1.5 & 2.0 & 2.5 \\
\hline 0.1 & 2.5 & 0.05 & 0.0 & $|q|$ & 0.750 & 0.642 & 0.488 & 0.339 & 0.220 & 0.135 \\
\hline & & & & $\theta$ & 0.0 & -.0324 & -.0563 & -.0759 & -.0932 & -.1089 \\
\hline & & & 1.0 & $|q|$ & 0.750 & 0.568 & 0.383 & 0.237 & 0.137 & 0.075 \\
\hline & & & & $\theta$ & 0.0 & -.0331 & -.0566 & -.0755 & -.0919 & -.1066 \\
\hline & & 0.1 & 1.0 & $|q|$ & 0.750 & 0.561 & 0.374 & 0.229 & 0.131 & 0.071 \\
\hline & & & & $\theta$ & 0.0 & -.0327 & -.0560 & -.0748 & -.0911 & -.1058 \\
\hline & 25 & 0.05 & 0.0 & $|q|$ & 0.5 & 0.336 & 0.261 & 0.234 & 0.225 & 0.218 \\
\hline & & & & $\theta$ & 0.0 & -.1001 & -.2307 & -.3494 & -.4356 & -.4994 \\
\hline & & & 1.0 & $|q|$ & 0.5 & 0.339 & 0.272 & 0.243 & 0.222 & 0.201 \\
\hline & & & & $\theta$ & 0.0 & -.1608 & -.3429 & -.4940 & -.6106 & -.7090 \\
\hline & & 0.1 & 1.0 & $|q|$ & 0.5 & 0.330 & 0.255 & 0.220 & 0.195 & 0.172 \\
\hline & & & & $\theta$ & 0.0 & -.1411 & -.3047 & -.4440 & -.5523 & -.6434 \\
\hline 1.0 & 2.5 & 0.05 & 0.0 & $|q|$ & 0.750 & 0.602 & 0.446 & 0.306 & 0.197 & 0.121 \\
\hline & & & & $\theta$ & 0.0 & -.3078 & -.5446 & -.7431 & -.9196 & -1.080 \\
\hline & & & 1.0 & $|q|$ & 0.750 & 0.528 & 0.346 & 0.211 & 0.121 & 0.066 \\
\hline & & & & $\theta$ & 0.0 & -.3103 & -.5439 & -.7355 & -.9032 & -1.055 \\
\hline & & 0.1 & 1.0 & $|q|$ & 0.750 & 0.522 & 0.339 & 0.205 & 0.117 & 0.063 \\
\hline & & & & $\theta$ & 0.0 & -.3062 & -.5378 & -.7283 & -.8954 & -1.047 \\
\hline & 25 & 0.05 & 0.0 & $|q|$ & 0.5 & 0.248 & 0.114 & 0.077 & 0.083 & 0.084 \\
\hline & & & & $\theta$ & 0.0 & -.2770 & -.7978 & -1.669 & -2.215 & -2.451 \\
\hline & & & 1.0 & $|q|$ & 0.5 & 0.208 & 0.083 & 0.060 & 0.057 & 0.047 \\
\hline & & & & $\theta$ & 0.0 & -.3188 & -.9945 & -1.909 & -2.343 & -2.530 \\
\hline & & 0.1 & 1.0 & $|q|$ & 0.5 & 0.208 & 0.082 & 0.057 & 0.053 & 0.044 \\
\hline & & & & $\theta$ & 0.0 & -.3118 & -.9621 & -1.871 & -2.316 & -2.603 \\
\hline
\end{tabular}

Table 1 - Magnitude $|q|$ and inclination $\theta$ of the fluid velocity vector near the plate for different values of $E, t, n, k$.

$E=0.1$ and $E=1.0$. The effects of the particles and the magnetic field are also considered in each case. It is seen that the drag on the plate fluctuates with time in a manner similar to that of pulses on the plate for all values of rotation. Moreover, the drag, in each case, becomes negative at the end and at the beginning of consecutive pulses acting on the plate. This is expected because near the end of the decelerating motion of the plate the fluid in motion drives it towards its destination by exerting drag in opposite direction (negative drag) which prevails till the plate acquires sufficient momentum to overcome the negative drag during its next accelerated motion. Besides, for small values of rotation, for instance, 
when $E=0.0$ and $E=0.1$, the maximum value of the drag is found to occur at about $t=2 \tau$ which for large rotation $(E=1.0)$ appears at about $t=6 \tau$. Similar is the case for the appearance of the minimum value of the drag on the plate. This shows that the time of occurrence of the maximum and the minimum of the drag on the plate depends on rotation. In general, the drag on the plate is increased by rotation, the magnetic field and the particles with the effect of rotation increased due to the particles and diminished due to the magnetic field. For instance, when $t=5.0, n=0.3$ and $E$ is increased from 0.1 to 1.0 , the drag on the plate increases by $0.242 \%$ and $0.338 \%$ for $k=0.4$ and 0.9 respectively. Similarly, for $t=5.0, k=0.4$ and $E$ is increased from 0.1 to 1.0 , the drag on the plate increases by $0.242 \%$ and $0.174 \%$ for $n=0.3$ and 0.5 respectively. Moreover, we find that the increasing effect of the particles on the drag diminishes due to increase of the magnetic field both in the rotating and non-rotating system. Finally, we notice that, for all values of rotation, the steady-state result (eqn. 5.3) contains the effect of both the particles and the magnetic field, which differs from that of impulsively moved plate, in which case, the steady-state result for the drag contains both the effects only when rotation is large [Ref. 11].

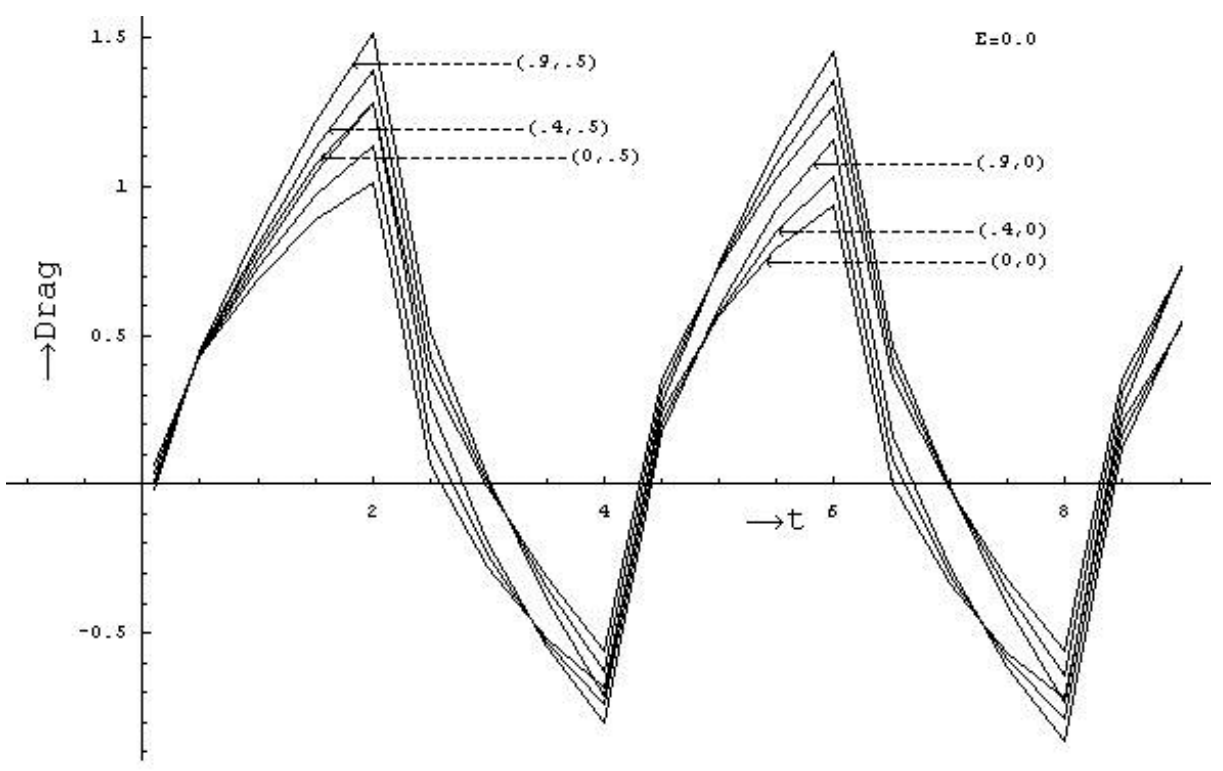

Figure 9 - Drag on the plate for various values of $(k, n)$ when $T=2$ and $E=0.0$. 


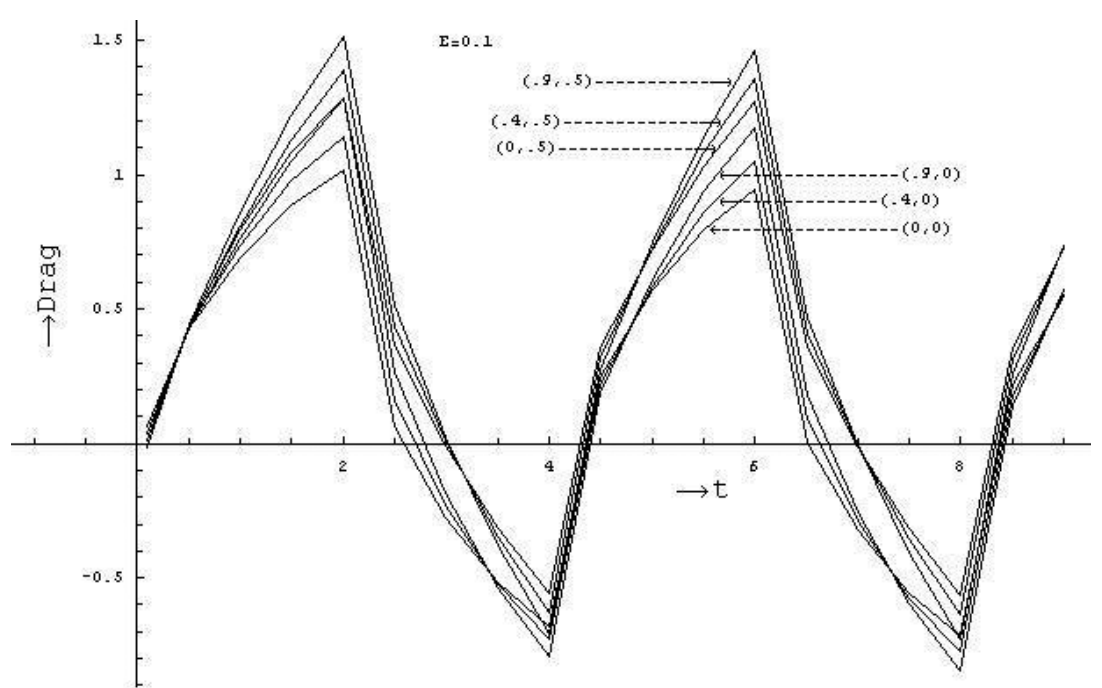

Figure 10(a) - Drag on the plate for various values of $(k, n)$ when $T=2$ and $E=0.1$.

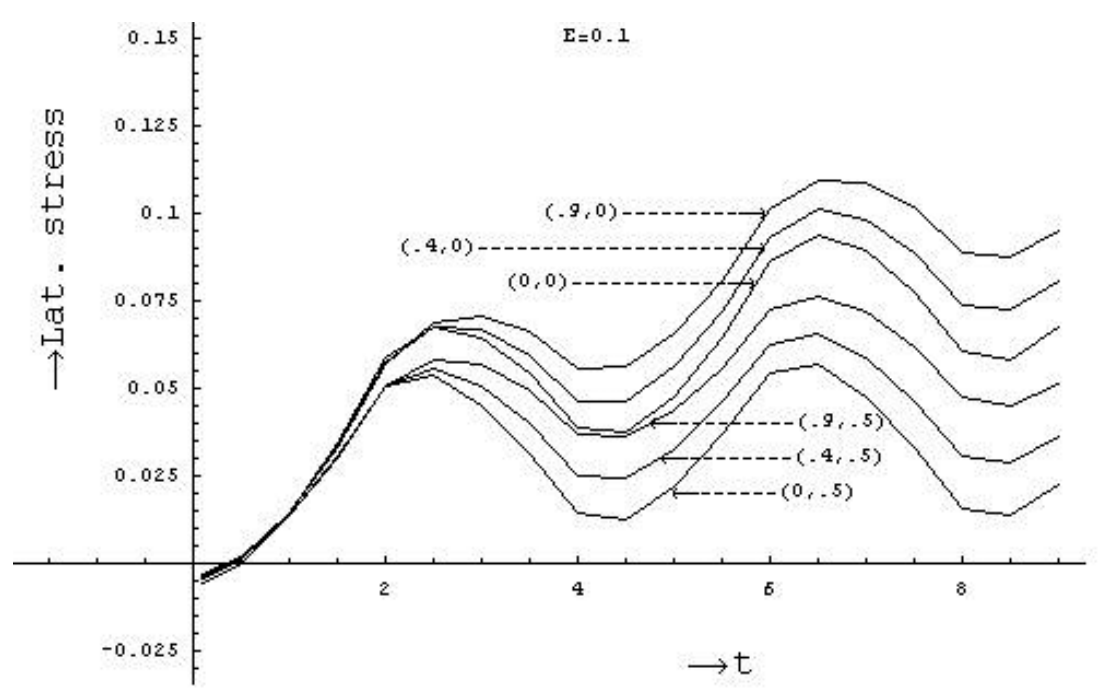

Figure 10(b) - Lateral stress on the plate for various values of $(k, n)$ when $T=2$ and $E=0.1$.

The lateral stress, on the other hand, developed due to rotation, fluctuates with time remaining always positive for all values of the flow parameters excepting at the initial stage of the motion, which is not similar to that of drag. Moreover, 


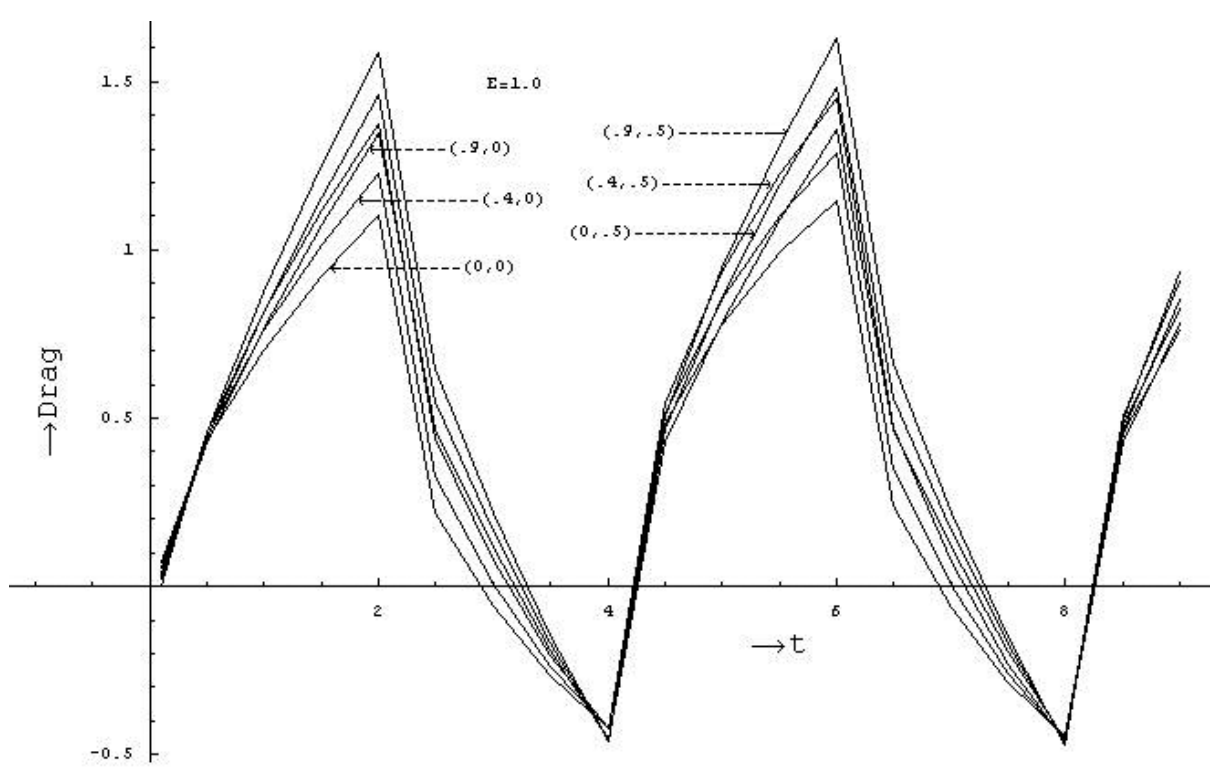

Figure 11(a) - Drag on the plate for various values of $(k, n)$ when $T=2$ and $E=1.0$.

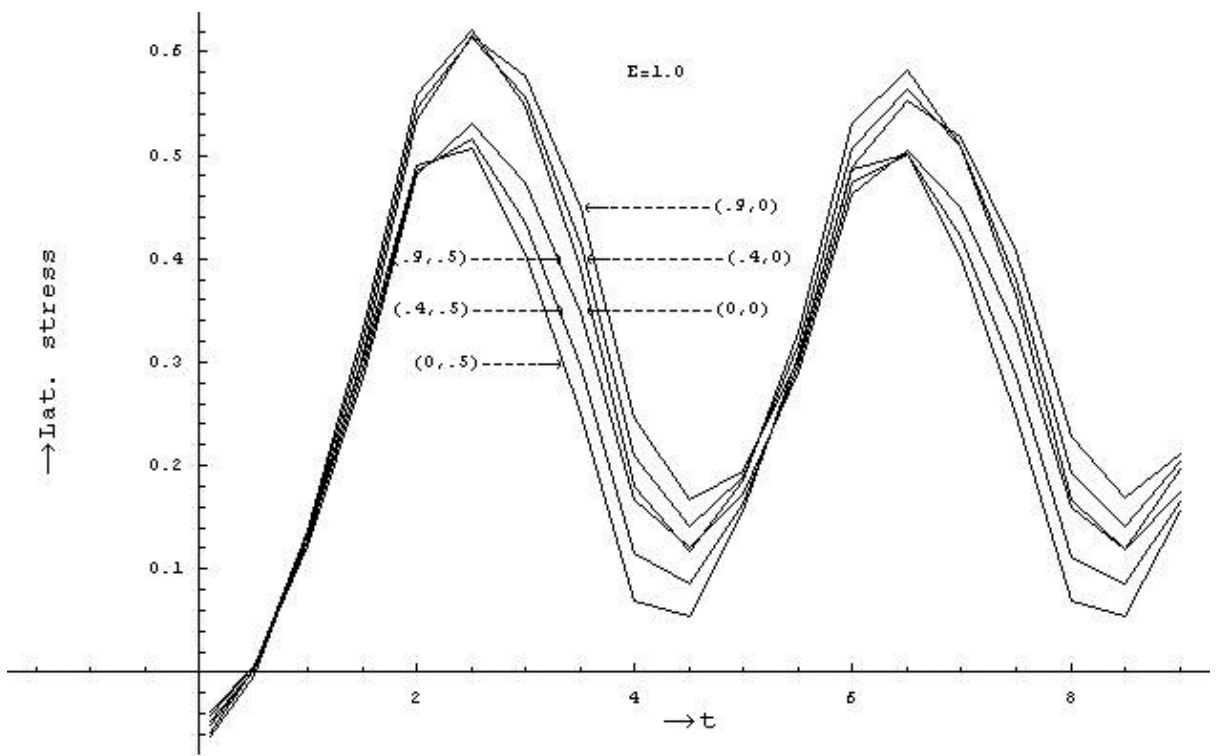

Figure 11(b) - Lateral stress on the plate for various values of $(k, n)$ when $T=2$ and $E=1.0$.

Comp. Appl. Math., Vol. 27, N. 1, 2008 
unlike drag, the magnitude of the lateral stress diminishes with the magnetic field and increases with the particles irrespective of the values of rotation. The occurrence of the maximum value of the lateral stress depends on rotation with its minimum at the initial stage of the motion. For instance, when $E=0.1$, the maximum of the lateral stress occurs at about $t=6.5 \tau$ and the same is found at about $t=2.5 \tau$ when $E=1.0$. Additionally, we observe that the increasing effect of rotation on the lateral stress increases with the magnetic field and decreases with the particles which is just reversed to that observed in the case of drag on the plate.

Finally, the magnitude and inclination of the fluid velocity vector with increasing $z$ and for different values of $E, t, n, k$ are shown in the Table I so that an understanding about the propagation of disturbance away from the plate and the structure of the associated Ekman spirals can be ascertained.

\section{Conclusion}

The Present problem is concerned with the investigation of the unsteady motion in a fluid-particle system induced by multiple frequency unidirectional motion of the boundary instead of the motion generated by oscillations of the boundary with constant frequency about a constant mean as considered by the authors [11]. Accordingly, the flow phenomena encountered by the present authors and the authors [11] are not the same. In the present investigation, the velocity distribution remains unidirectional and positive for all the time instead of oscillating positive and negative flows as obtained by the authors [11]. However, the present findings coincide with those of authors [11] only in the case of an impulsively moved plate as it represents the same motion. It is further to be noted that blood flows in large arteries, some atmospheric and oceanic flows driven by pressure pulses and other similar problems associated with astrophysics, which are generally considered as unidirectional pulsatile flows, may easily be investigated following the present analysis instead of that given by authors [11].

Additionally, as the major findings of the present analysis we notice that (i) in presence of pulsation the particles increases the flow near the plate only when rotation is small and time is large. Such a phenomenon does not occur in presence of pulsation with large rotation or in absence of pulsation with any value of 
rotation, (ii) the intensity of pulsatile flow near the plate remains always less than the impulsively generated flow, (iii) the magnetic field and the particles require some time to exert their influence on the drag and the lateral stress from the beginning of the unsteady motion of the plate caused by the pulses. This is true for all values of rotation, (iv) for all values of rotation, the magnitude of drag is increased by both the magnetic field and the particles while the magnitude of the lateral stress is decreased by the magnetic field and increased by the particles.

Acknowledgement. The authors are grateful to the referees for providing many useful suggestions to improve the paper in its present form.

\section{REFERENCES}

[1] A.K. Ghosh, Flow of viscous liquid set up between two co-axial cylinders due to pulses of longitudinal impulses applied on the inner cylinder. J. Sci. Engg. Res., 9(2) (1965), 222.

[2] A. Chakraborty and J. Ray, Unsteady magnetohydrodynamic Couette flow between two plates when one of the plates is subjected to random pulses. J. Phys. Soc. Jpn., 48 (1980), 1361.

[3] M.N. Makar, Magnetohydrodynamic flow between two plates when one of the plates is sujected to tooth pulses. Acta Phys. Pol., A71 (1987), 995.

[4] A.R. Bestman and F.I. Njoku, On hydromagnetic channel flow induced by tooth pulses. Preprint IC/88/10, Miramare-Trieste, (1988).

[5] N. Datta, D.C. Dalal and S.K. Misra, Unstesdy heat transfer to pulsatile flow of a dusty viscous incompressible fluid in a channel. Int. J. Heat Mass Transfer, 36(7) (1993), 1783.

[6] N. Datta and D.C. Dalal, Pulsatile flow and heat transfer of a dusty fluid through an infinitely long annular pipe. Int. Multiphase Flow, 21(3) (1995), 515.

[7] A.K. Ghosh and K. Sarkar, On hydromagnetic channel flow of a dusty fluid induced by tooth pulses. J. Phys. Soc. Jpn., 64(3) (1995), 3001.

[8] A.K. Ghosh and L. Debnath, On hydromagnetic pulsatile flow of a two-phase fluid. ZAMM, 76(2) (1996), 121.

[9] S. Ghosh and A.K. Ghosh, On hydrmagnetic channel flow of a particulate suspension induced by rectified sine pulses. J. Phys. Soc. Jpn., 73(6) (2004), 1506.

[10] S. Ghosh and A.K. Ghosh, On hydromagnetic flow of a two-phase fluid near a pulsating plate. Indian J. Pure. Appl. Math., 36(10) (2005), 529.

[11] A.K. Ghosh and L. Debnath, Hydromagnetic Stokes flow in a rotating fluid with suspended small particles. Appl. Sci. Res., 43 (1986), 165. 
[12] A.K. Ghosh and L. Debnath, On hydrodynamic rotating flow of a two-phase fluid. ZAMM, 75(2) (1995), 156.

[13] H.P. Greenspan, The theory of rotating fluids. Camb. Univ. Press, (1969).

[14] R. Hide and P.H. Roberts, The origin of the mean geomagnetic field. In: Physics and Chemistry of the Earth, vol. 4, Pergamon Press, New York, (1961).

[15] R.H. Dieke, Internal rotation of the sun. In: L. Goldberg (Ed.). Annual Review of Astronomy and Astrophysics, vol. 8, Annual Reviews Inc. (1970).

[16] M. Kumari and G. Nath, Unsteady MHD film flow over a rotating infinite disk. Int. J. Engg. Sci., 42 (2004), 1099.

[17] P.G. Saffmam, On the stability of laminar flow of a dusty gas. J. Fluid Mech., 13 (1962), 120.

[18] C. Thornley, On Stokes and Rayleigh layers in a rotating system. Quart. J. Mech. and Appl. Math., 21 (1968), 451.

[19] H.T. Yang and J.V. Healy, The Stokes Problems for a conducting fluid with a suspension of particles. Appl. Sci. Res., 21 (1973), 387.

[20] S.I. Pai, Magnetogasdynamics and Plasma Dynamics. Prentice Hall, Inc., Englewood Cliffs, New Jersey, 1962.

[21] D.H. Michael and D.A. Miller, Plane parallel flow of a dusty gas. Mathematka, 13 (1966), 97. 